\title{
Physical and Mechanical Properties of Ni-Cr based composites with addition of solid lubricants produced through powder metallurgy process
}

\author{
Wan Farhana Mohamad ${ }^{1, a}$, Amir Azam Khan ${ }^{1}$, Faiz Ahmad $^{2}$ and Abdullah Yassin ${ }^{1}$ \\ ${ }^{1}$ Department of Mechanical and Manufacturing, Faculty of Engineering, Universiti Malaysia Sarawak, 94300 Kota Samarahan, Sarawak, \\ Malaysia \\ ${ }^{2}$ Mechanical Engineering Department, University Technology PETRONAS, 31750 Tronoh, Perak, Malaysia
}

\begin{abstract}
Ni-Cr based composites with and without the addition of solid lubricants $\left(\mathrm{MoS}_{2}\right.$, silver and $\left.\mathrm{CaF}_{2}\right)$ were prepared by powder metallurgy method. The samples were sintered at two different temperatures, $1000{ }^{\circ} \mathrm{C}$ and 1200 ${ }^{\circ} \mathrm{C}$. The physical properties such as shrinkage, sintered density and porosity were studied. The microstructures of the $\mathrm{Ni}-\mathrm{Cr}$ based composites were observed by using SEM analysis while the mechanical properties of the composites were measured by Rockwell Hardness Tester. The results revealed that the increased in sintering temperature improved the shrinkage, sintered density and hardness of the composites while less porosity produced. Ni-Cr based composites with the addition of silver and $\mathrm{MoS}_{2}$ exhibited better shrinkage, density and porosity. Besides, $5 \%$ of $\mathrm{MoS}_{2}$ addition in the composites improves the hardness of the composites at sintering temperature $1200^{\circ} \mathrm{C}$.

Keywords: solid lubricants, $\mathrm{Ni}-\mathrm{Cr}$ composites, powder metallurgy, $\mathrm{MoS}_{2}$, silver, $\mathrm{CaF}_{2}$
\end{abstract}

\section{Introduction}

Giant industries such as automotive and aerospace are having an economical loss due to high maintenance cost for mechanical components. Failure of mechanical components such as bearings and bushings used in the advanced jet engines are caused by friction and wear as subjected to wide temperature range. As for example, the operation temperature of nozzles for turbojet propulsion system is reaching the temperature of $1650^{\circ} \mathrm{C}$ [1]. At this high temperature, the liquid lubricant is unstable and tends to lose its lubricating properties. Thus, solid lubricant is preferable compared to the liquid lubricant. Besides, solid lubricant also has advantages compared to liquid lubricants either under the extreme pressure conditions, radiation environment or cryogenic temperature [2].

The incorporation of the solid lubricant in the composites is called self-lubricating composites. The composite is able to form a lubricating film to reduce the effect of friction and wear. The examples of metal used in the self-lubricating composites are iron based, copper based and nickel based composites. Iron based selflubricating composites are mostly used in the automotive application such as piston ring, clutch, brake system and engine liners [3]. While copper based composites are used for the application of thermal management application due to excellent properties thermal and heat conductivity [4]. Among the metal composites, nickel based composites have become the most attention of researchers for high temperature application. The high cost of refractory metals and complex manufacturing process also make the metal nickel as an option for high temperature application [5].

Nickel Chromium (Ni-Cr) has become one of the leading materials for high temperature application due to its excellent performance at high temperature. A series of nickel based composites has been developed in order to achieve the great need of high temperature solid lubricating system produced through powder metallurgy process [6]. Ni-Cr matrix also acted as a binder and offered excellent high temperature oxidation/corrosion resistance and essential mechanical strength [7], [8]. Nickel itself offers good mechanical properties and antioxidizing properties when exposed to air atmosphere at high temperature while chromium offers anti-wear and lubricating properties at high temperature [9]. The strength of the $\mathrm{Cr}$ particles can determine the strength and bonding between the matrix and $\mathrm{Cr}$ reinforcement.

Efforts have been made in order to enhance the performance of self-lubricating composites with solid lubricants addition. The work is continued in order to develop a perfect combination of matrix and solid lubricant for high temperature system to meet the requirement of advanced technology. In this research work, the author is working on $\mathrm{Ni}-\mathrm{Cr}$ based composites with the addition of single, dual and multiple solid lubricants in order to obtain an excellent mechanical as

\footnotetext{
a Corresponding author: wanfarhana88@gmail.com
} 
well as tribological properties. The composites are fabricated by powder metallurgy method.

\section{Experimental}

The Nickel -Chromium (Ni-Cr) composites are based on $80 \% \mathrm{Ni}$ and $20 \% \mathrm{Cr}$. The solid lubricants added are molybdenum disulphide $\left(\mathrm{MoS}_{2}\right)$, silver $(\mathrm{Ag})$ and calcium fluoride $\left(\mathrm{CaF}_{2}\right)$. All of the raw materials are supplied by Robert Scientific Sdn Bhd. The characterizations of powders were done using Scanning Electron Microscope (SEM) by Hitachi Tabletop TM3030 and X-Ray Fluorescences (XRF) by Bruker S4 Pioneer, USA.

\subsection{Preparation of samples}

\subsubsection{Samples preparations}

$\mathrm{Ni}-\mathrm{Cr}$ based composites have been produced by powder metallurgy method which consists of mixing, compaction and sintering. NC denoted for $\mathrm{Ni}-\mathrm{Cr}$ based composite (without solid lubricant), NCM (with $\mathrm{MoS}_{2}$ ), NCA (with $\mathrm{Ag}$ ), NCCf (with $\mathrm{CaF}_{2}$ ), NCMA (with $\mathrm{MoS}_{2}$ and $\mathrm{Ag}$ ), NCMCf (with $\mathrm{MoS}_{2}$ and $\mathrm{CaF}_{2}$ ), NCACf (with Ag and $\mathrm{CaF}_{2}$ ) and NCMACf (with $\mathrm{MoS}_{2}, \mathrm{Ag}$ and $\mathrm{CaF}_{2}$ ). The powder has been weighted by analytical balance based on composition in Table 1 . The powder mixture was mixed homogeneously in a ball mill for 30 minutes with the speed of $300 \mathrm{rpm}$. A mixture of powder was compacted using compression machine with the pressure of $100 \mathrm{kN}$ at room temperature. The compacted samples were in a pellet shape with the dimension of $13 \mathrm{~mm} \times 5 \mathrm{~mm}$. The compacted samples were sintered in a high temperature furnace for 60 minutes. The sintering atmosphere was argon gas with a flow rate of $4 \mathrm{~L} / \mathrm{min}$. The compacted samples were sintered at the temperature of $1000{ }^{\circ} \mathrm{C}$ and $1200{ }^{\circ} \mathrm{C}$ with a heating rate of $10^{\circ} \mathrm{C} / \mathrm{min}$.

Table 1. Materials compositions for Ni-Cr based composites.

\begin{tabular}{|l|l|c|c|c|c|}
\hline \multirow{2}{*}{ Samples } & \multirow{2}{*}{ Designation } & \multicolumn{4}{|c|}{ Composition (wt\%) } \\
\cline { 3 - 6 } & & $\begin{array}{c}\mathbf{8 0 N i}- \\
\mathbf{2 0 C r}\end{array}$ & $\mathbf{M o S}_{\mathbf{2}}$ & $\mathbf{A g}$ & $\mathbf{C a F}_{\mathbf{2}}$ \\
\hline 1 (Pure) & NC & Balance & 0 & 0 & 0 \\
\hline 2 (Single) & NCM & Balance & 5 & 0 & 0 \\
\hline 3 (Single) & NCA & Balance & 0 & 5 & 0 \\
\hline 4 (Single) & NCCf & Balance & 0 & 0 & 5 \\
\hline 5 (Dual) & NCMA & Balance & 5 & 5 & 0 \\
\hline 6 (Dual) & NCMCf & Balance & 5 & 0 & 5 \\
\hline 7 (Dual) & NCACf & Balance & 0 & 5 & 5 \\
\hline 8 (Multiple) & NCMACf & Balance & 5 & 5 & 5 \\
\hline
\end{tabular}

\subsection{Physical Properties}

The diameter and height of the sintered samples were measured by using vernier caliper while the mass of the samples was obtained by analytical balance. At least 3 measurements were taken and the average value was measured. The shrinkage, density, and porosity of the samples were calculated using the geometric method (formula).

\subsubsection{Shrinkage}

The volume of the green and sintered samples in pellet size are calculated by using the formula $V=\pi(d / 2)^{2} h$. The percentage of shrinkage is measured by using formula :

$$
\begin{gathered}
\% \text { of shrinkage }=\left(V_{1}-V_{2}\right) /\left(V_{1}\right) \times 100 \\
V_{1}=\text { Volume before sintering } \\
V_{2}=\text { Volume after sintering }
\end{gathered}
$$

The measurements of height (h) and diameter (d) were taken 3 times to get the average value.

\subsubsection{Density}

The density of green and sintered samples is measured before and after sintering. The density, $\rho$ formula is :

$$
\rho=m / V
$$

$$
m=\text { mass of pellet }(\mathrm{g})
$$

$$
V=\text { Volume of pellet }\left(\mathrm{cm}^{3}\right)
$$

\subsubsection{Porosity}

The porosity of the composites was measured by formula of:

$$
\begin{gathered}
\text { Porosity }=\left(\rho_{\text {theoritical }}-\rho_{\text {sintered }}\right) /\left(\rho_{\text {theoritical }}\right) \times 100 \\
\rho_{\text {theoritical }}=\text { theoretical density } \\
\rho_{\text {sintered }}=\text { sintered density }
\end{gathered}
$$




\subsection{Microstructure and Mechanical Properties}

\subsubsection{Microstructure}

The microstructure of the sintered samples was observed and analyzed by using Scanning Electron Microscope (SEM). The samples were ground with silicon carbide paper and polished with diamond paste as a surface preparation before SEM analysis. The effect of sintering temperature and composition of the composites were studied.

\subsubsection{Hardness}

The hardness of Ni-Cr based composites was measured by using Mitutoyo Hardness Machine with the diamond indenter at an indentation load of $1471 \mathrm{~N}$. At least 10 measurements were taken and the average value is calculated.

\section{Results and Discussions}

\subsection{Characterizations of raw materials}

SEM analysis was done to observe the particle shape and distribution while XRF analysis was conducted to determine the purity of the powder. Figure 1 and 2 show the SEM micrograph of the nickel and chromium powder.

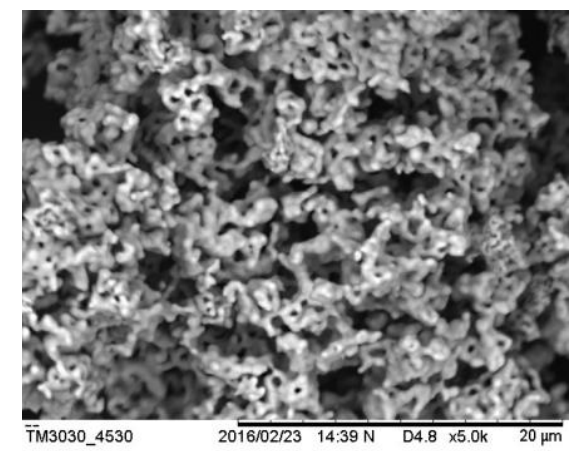

Figure 1. SEM microstructure of nickel powder at a magnification of 5000X.

In Figure 1, SEM analysis with a magnification of 5000X illustrates the nickel powder used in this research. The purity of the nickel powder is $99.1 \%$ determined by XRF analysis.

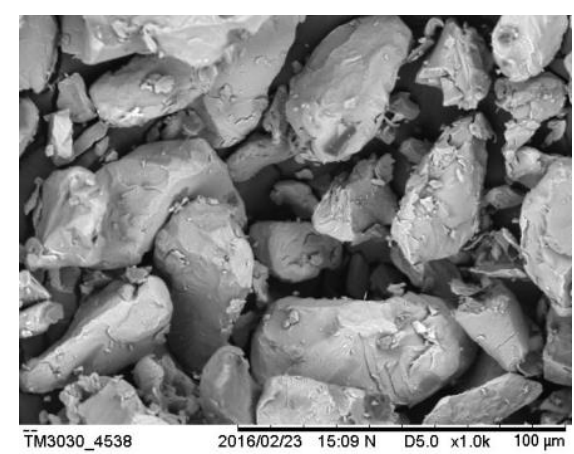

Figure 2. SEM microstructure of chromium powder at a magnification of $1000 \mathrm{X}$.
Figure 2 shows the particle shape and distribution of chromium powder. The particle shape is in the irregular shape. XRF analysis determines the purity of chromium powder is $93.3 \%$. Figure 3-5 shows the SEM microstructure of solid lubricants which are $\mathrm{MoS}_{2}$, silver and $\mathrm{CaF}_{2}$.

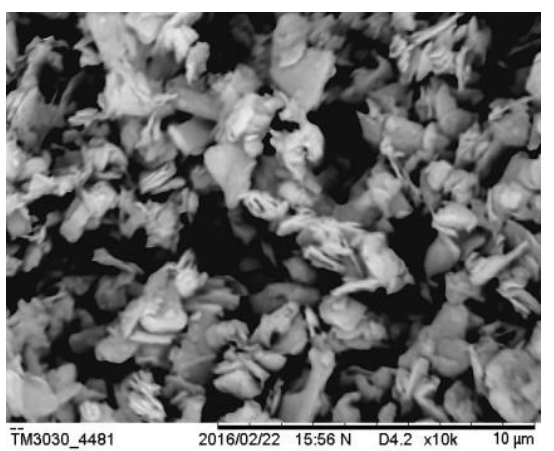

Figure 3. SEM microstructure of $\mathrm{MoS}_{2}$ powder at a magnification of $10000 \mathrm{X}$.

From the Figure 3, it demonstrates the particle shape of $\mathrm{MoS}_{2}$ powder which is in flake shape. The purity of the powder is Mo $72 \%$ and $\mathrm{S} 25.1 \%$.

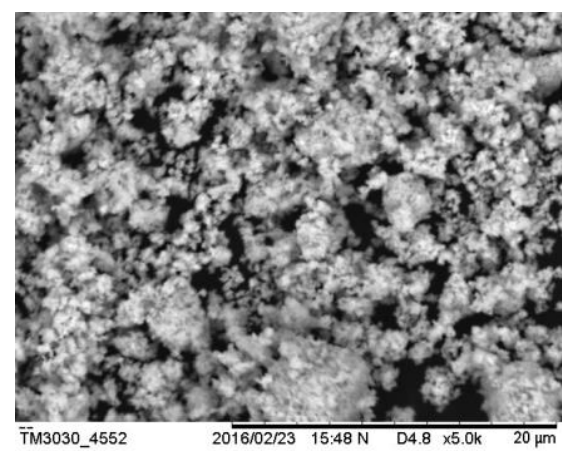

Figure 4. SEM microstructure of silver powder at a magnification of $5000 \mathrm{X}$.

SEM microstructure in Figure 4 shows the particle distribution of silver powder used in the research work.

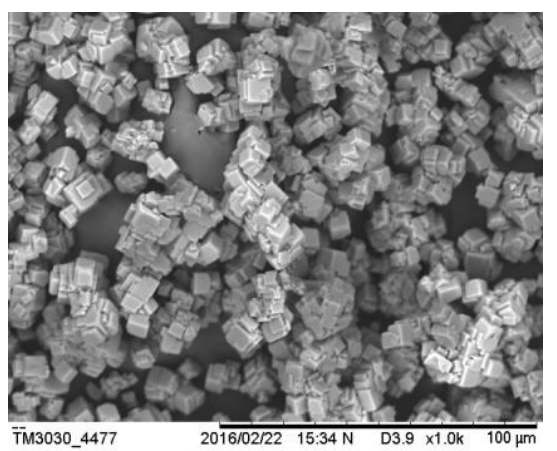

Figure 5. SEM microstructure of $\mathrm{CaF}_{2}$ powder at a magnification of $1000 \mathrm{X}$.

Based on Figure 5, the micrograph illustrates the stacked crystallize structure of $\mathrm{CaF}_{2}$. The microstructure in cubic particle shape. The purity of powder is $\mathrm{Ca} 99.1 \%$. 


\subsection{Characterization of mixed powders.}

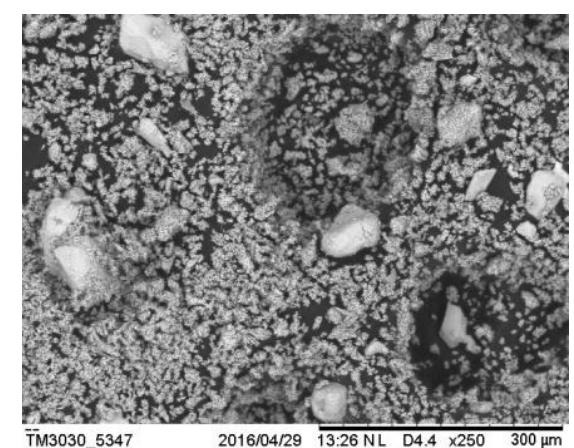

Figure 6. A mixture of $\mathrm{Ni}$ and $\mathrm{Cr}$ powders after 30 minutes of ball milling.

Figure 6 shows the distribution of Nickel and Chromium powder after mixing by using ball mill machine for 30 minutes. The bigger particles represent chromium powder while the smaller particles represent nickel powder. The mixture was homogeneously distributed throughout the process. Some smaller particles of nickel tend to agglomerate on the chromium surface. After that, the mixture was compacted by a hydraulic press into a pallet shape. The compacted samples were sintered in the tube furnace at the sintering temperature of $1000{ }^{\circ} \mathrm{C}$ and 1200 ${ }^{\circ} \mathrm{C}$ for 60 minutes in the argon atmosphere.

\subsection{Shrinkage of Ni-Cr based composites}

After sintering at high temperature, the compacted samples were subjected to shrinkage. There was a reduction in the volume of compacted samples. Table 2 demonstrates the percentage of shrinkage after sintering at different temperatures. As the sintering temperature increased from 1000 to $1200{ }^{\circ} \mathrm{C}$, the shrinkage of the samples also increased.

Table 2. Percentage of Shrinkage after sintering at $1000{ }^{\circ} \mathrm{C}$ and $1200{ }^{\circ} \mathrm{C}$.

\begin{tabular}{|l|c|c|}
\hline \multirow{2}{*}{ Samples } & \multicolumn{2}{|c|}{ Shrinkage (\%) } \\
\cline { 2 - 3 } & at $1000{ }^{\circ} \mathrm{C}$ & at $1200{ }^{\circ} \mathrm{C}$ \\
\hline NC & 15.56 & 32.47 \\
\hline NCM & 21.65 & 35.07 \\
\hline NCA & 25.14 & 38.18 \\
\hline NCCf & 24.90 & 34.77 \\
\hline NCMA & 24.83 & 37.55 \\
\hline NCMCf & 22.09 & 33.79 \\
\hline NCACf & 24.35 & 33.61 \\
\hline NCMACf & 20.92 & 37.50 \\
\hline
\end{tabular}

Based on Table 2, the highest percentage of shrinkage for both temperatures is NCA composites for $25.14 \%$ (1000 ${ }^{\circ} \mathrm{C}$ ) and $38.18 \%$ (at $1200{ }^{\circ} \mathrm{C}$ ) while the lowest percentage of shrinkage for both temperatures is $\mathrm{NC}$ composites for $15.56 \%\left(1000{ }^{\circ} \mathrm{C}\right.$ ) and $32.47 \%$ (at $1200{ }^{\circ} \mathrm{C}$ ). During sintering, the center to center distance between the powder particles is reducing and at the same time, the pore shrinks [10]. The addition of solid lubricant/s increased the percentage of shrinkage of the Ni-Cr based composites. The highest shrinkage were NCA composites at both temperatures. This was due to the smaller particles of silver which filled in the pores and reduced the size of pores. Shrinkage is one of the factors to the increment of sintered density.

\subsection{Sintered Density of $\mathrm{Ni}-\mathrm{Cr}$ based composites}

Shrinkage of the composites leads to the densification of the composites. Sintered density is the measurement result for densification. Table 3 illustrates the sintered density of $\mathrm{Ni}-\mathrm{Cr}$ based composites samples at sintering temperature of $1000{ }^{\circ} \mathrm{C}$ and $1200{ }^{\circ} \mathrm{C}$. Sintered density was also enhanced from the sintering temperature of 1000 to $1200{ }^{\circ} \mathrm{C}$.

Table 3. Sintered density of $\mathrm{Ni}-\mathrm{Cr}$ based composites samples at sintering temperature of $1000{ }^{\circ} \mathrm{C}$ and $1200{ }^{\circ} \mathrm{C}$.

\begin{tabular}{|l|c|c|}
\hline \multirow{2}{*}{ Samples } & \multicolumn{2}{|c|}{ Sintered Density $\left(\mathrm{g} / \mathrm{cm}^{3}\right)$} \\
\cline { 2 - 3 } & at $1000{ }^{\circ} \mathrm{C}$ & at $1200{ }^{\circ} \mathrm{C}$ \\
\hline NC & 5.59 & 6.99 \\
\hline NCM & 5.67 & 7.08 \\
\hline NCA & 6.07 & 7.36 \\
\hline NCCf & 5.91 & 6.67 \\
\hline NCMA & 6.15 & 7.23 \\
\hline NCMCf & 5.58 & 6.54 \\
\hline NCACf & 6.08 & 6.94 \\
\hline NCMACf & 5.57 & 6.88 \\
\hline
\end{tabular}

From Table 3, the highest sintered density is achieved by NCA composite followed by NCMA composites at sintering temperature $1200{ }^{\circ} \mathrm{C}$. Increased in density demonstrate that the process of diffusion, densification, recrystallization and grain growth between the particles at the contact area. The result is supported by the percent density graph as shown in Figure 7.

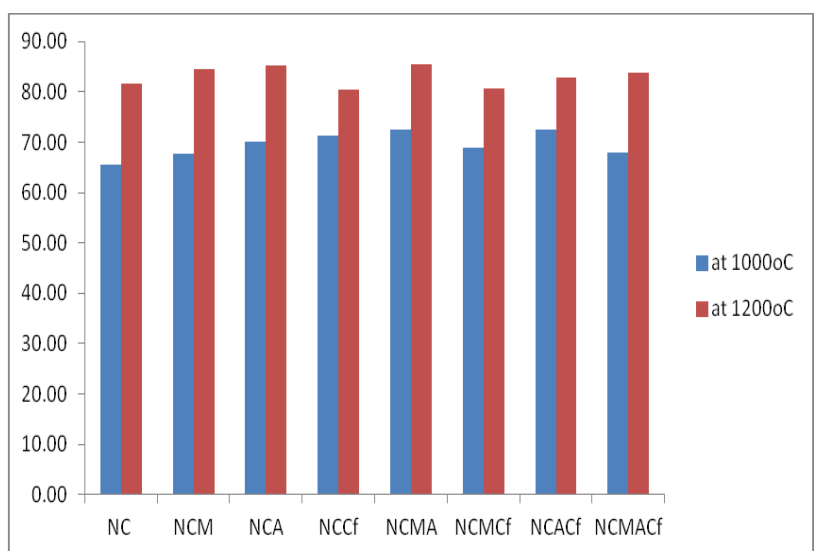

Figure 7. The percent density of Ni-Cr based composites at sintering temperature of 1000 and $1200{ }^{\circ} \mathrm{C}$.

The percent density indicated the densification or pore shrinkage of the composites. During sintering, the particles are diffusing into each other as the temperature rise. Based on Figure 7, the highest percent density was achieved by NCMA followed by NCA and NCM composites at sintering temperature of $1200{ }^{\circ} \mathrm{C}$. Small and fine size of solid lubricant which was silver and 
$\mathrm{MoS}_{2}$ compared to $\mathrm{CaF}_{2}$ help in the diffusion and densification process during sintering. Then, densification leads to decrease in porosity and increase the particle contact area.

\subsection{Porosity in $\mathrm{Ni}-\mathrm{Cr}$ based composites}

As increasing the sintering temperature, the porosity of the Ni-Cr based composites is decreasing. The number of porosities is decreased and the size of pores are reduced. When a higher temperature is applied, the grain of the composite will grow bigger and the pores tend to shrink. Thus, the porosity of the composites is reduced [11]. The reason is the driving force of sintering is increasing when the sintering temperature rises. At the earlier stage of sintering, the driving force is produced by the surface energy which is associated with the internal surface area of the particles. This is where the grains of the composites grow bigger and the pore shrinks. Less porosity is desirable because it will contribute to the positive effect on mechanical properties. Table 4 shows the porosity in the $\mathrm{Ni}-\mathrm{Cr}$ based composites.

Table 4. Porosity in the Ni-Cr based composites samples after sintering at a temperature of $1000^{\circ} \mathrm{C}$ and $1200^{\circ} \mathrm{C}$.

\begin{tabular}{|l|c|c|}
\hline \multirow{2}{*}{ Samples } & \multicolumn{2}{|c|}{ Porosity $(\%)$} \\
\cline { 2 - 3 } & at $1000{ }^{\circ} \mathrm{C}$ & at $1200{ }^{\circ} \mathrm{C}$ \\
\hline NC & 35 & 18 \\
\hline NCM & 32 & 16 \\
\hline NCA & 30 & 15 \\
\hline NCCf & 29 & 19 \\
\hline NCMA & 27 & 15 \\
\hline NCMCf & 31 & 19 \\
\hline NCACf & 27 & 17 \\
\hline NCMACf & 32 & 16 \\
\hline
\end{tabular}

From Table 4, the lowest porosity is achieved by NCMA composites at both sintering temperature. This is due to the particle size of $\mathrm{MoS}_{2}$ and $\mathrm{Ag}$ which is smaller compared to $\mathrm{CaF}_{2}$. The particles of $\mathrm{MoS}_{2}$ and $\mathrm{Ag}$ tend to fill in the pores and eventually reduce the size of the pores. The porosity results can be supported by the SEM results in Figures 8-13. The black areas which represent porosity are decreasing as the sintering temperature increased from 1000 to $1200{ }^{\circ} \mathrm{C}$.

\subsection{Microstructure of $\mathrm{Ni}-\mathrm{Cr}$ based composites}

Figure 8 and 9 show the microstructure of Ni-Cr based composites without any addition of solid lubricants after sintering at $1000{ }^{\circ} \mathrm{C}$ and $1200{ }^{\circ} \mathrm{C}$. The grey phase represents the Nickel matrix while the darker phase represents the Chromium phase. On the other hand, the darkest (black) phase represent the porosity in the composite. More and larger size of pores could be seen in Figure 8 compared to Figure 9. When sintering at a higher temperature, the percentage of shrinkage is bigger, thus, reduce the distance between the particles and shrink the size of pores.

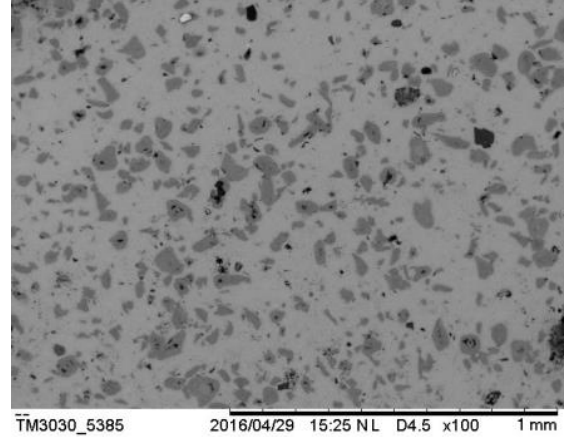

Figure 8. SEM micrograph of NC composite after sintering at $1000{ }^{\circ} \mathrm{C}$.

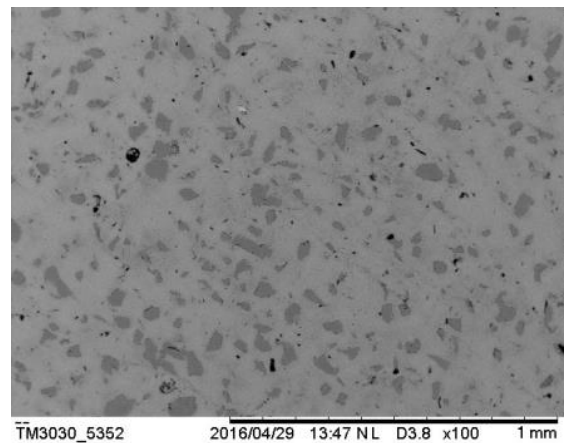

Figure 9. SEM micrograph of $\mathrm{NC}$ composite after sintering at $1200{ }^{\circ} \mathrm{C}$.

Figure 10 and 11 show the Ni-Cr based composites with the addition of silver as a solid lubricant. The existence of white phase represents the silver addition in the composite.

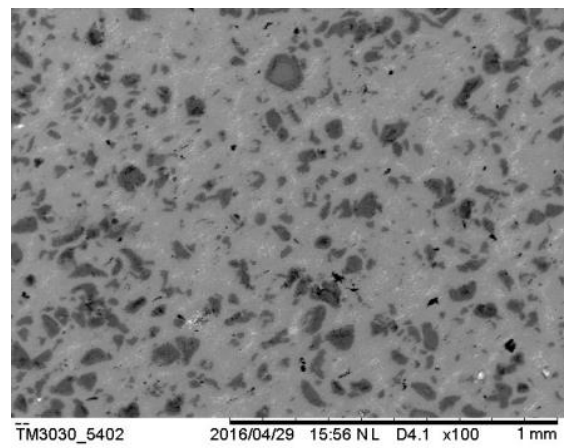

Figure 10. SEM micrograph of NCA composite after sintering at $1000{ }^{\circ} \mathrm{C}$.

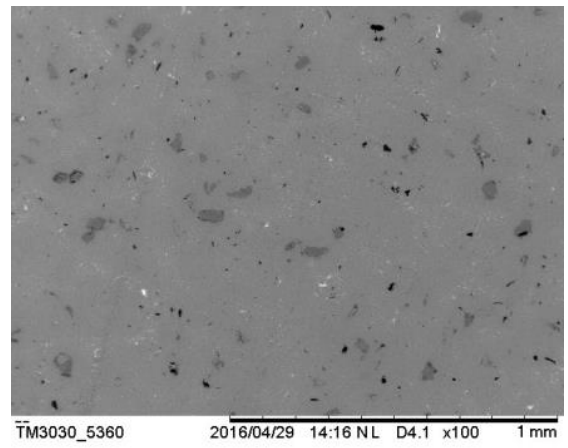

Figure 11. SEM micrograph of NCA composite after sintering at $1200{ }^{\circ} \mathrm{C}$. 
Figure 12 and 13 demonstrate the microstructure of Ni-Cr based composites with the addition of dual solid lubricant which are $\mathrm{MoS}_{2}$ and silver.

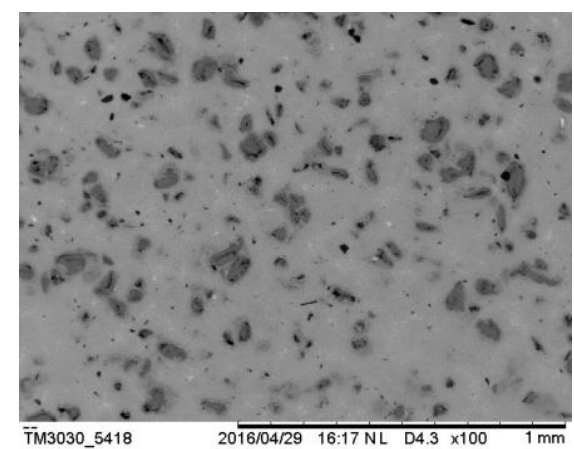

Figure 12. SEM microstructure of NCMA composites sintering at $1000{ }^{\circ} \mathrm{C}$.

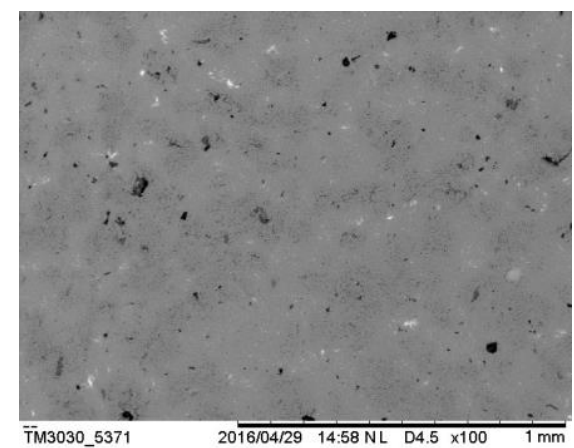

Figure 13. SEM microstructure of NCMA composites sintering at $1200{ }^{\circ} \mathrm{C}$.

\subsection{Hardness of $\mathrm{Ni}-\mathrm{Cr}$ based composites}

Higher shrinkage, enhancement of density and reduction of porosity will contribute to better mechanical properties. The hardness of the composites were listed in Table 5.

Table 5. The hardness of Ni-Cr based composites samples after sintering at temperature of $1000{ }^{\circ} \mathrm{C}$ and $1200{ }^{\circ} \mathrm{C}$.

\begin{tabular}{|l|c|c|}
\hline \multirow{2}{*}{ Samples } & \multicolumn{2}{|c|}{ Hardness (HRC) } \\
\cline { 2 - 3 } & at $1000{ }^{\circ} \mathrm{C}$ & at $1200{ }^{\circ} \mathrm{C}$ \\
\hline NC & 42.6 & 51.5 \\
\hline NCM & 36.8 & 52.1 \\
\hline NCA & 35.1 & 48.5 \\
\hline NCCf & 37.5 & 44.0 \\
\hline NCMA & 41.9 & 57.1 \\
\hline NCMCf & 35.8 & 46.4 \\
\hline NCACf & 36.0 & 44.8 \\
\hline NCMACf & 32.0 & 57.5 \\
\hline
\end{tabular}

Based on Table 5, at sintering temperature of $1000{ }^{\circ} \mathrm{C}$, the highest hardness is attained by the Ni-Cr composites. As we added the solid lubricants, the hardness of all Ni$\mathrm{Cr}$ based composites is decreasing. However, at a higher sintering temperature of $1200{ }^{\circ} \mathrm{C}$, the hardness of all of the composites containing $\mathrm{MoS}_{2}$ are increasing (NCM, NCMA and NCMACf). 5\% addition of $\mathrm{MoS}_{2}$ as solid lubricant helps in improving the mechanical properties of the composite. A suitable amount of solid lubricant addition is important in order to enhance mechanical properties of the composites [12].

The highest hardness achieved by NCMACf composites after sintering at $1200{ }^{\circ} \mathrm{C}$ with a value of $57.5 \mathrm{HRC}$. The lowest hardness also achieved by NCMACf composites after sintering at $1000{ }^{\circ} \mathrm{C}$ with a value of $32 \mathrm{HRC}$. The main reason is the sintering temperature play the main role in the reinforcement and strengthening of the composites. At sintering temperature of $1000{ }^{\circ} \mathrm{C}$, the addition of solid lubricant such as $\mathrm{MoS}_{2}, \mathrm{Ag}$ and $\mathrm{CaF}_{2}$ is not fully forming a good bonding of grain boundary. Thus, the grain growth between the particles is less thus the strengthening of the particles is also low compared to sintering at a higher temperature $\left(1200{ }^{\circ} \mathrm{C}\right)$ [13].

\section{Conclusions}

1. The increment in sintering temperature from $1000{ }^{\circ} \mathrm{C}$ to $1200{ }^{\circ} \mathrm{C}$ enhanced the shrinkage, density and hardness while reduced the porosity of $\mathrm{Ni}-\mathrm{Cr}$ based composites.

2. Ni-Cr based composites which contain silver and $\mathrm{MoS}_{2}$ (NCA and NCMA) achieved better physical properties such as shrinkage, density and porosity due the fine size of these solid lubricants compared to $\mathrm{CaF}_{2}$. The fine size of solid lubricant helped in filling the pores and thus increased the densification of the composites.

3. The mechanical properties of $\mathrm{Ni}-\mathrm{Cr}$ based composites are improving with the increasing sintering temperature and addition of $\mathrm{MoS}_{2}$. The addition of $5 \% \mathrm{MoS}_{2}$ in $\mathrm{Ni}-\mathrm{Cr}$ based composites improved their hardness. A suitable amount of solid lubricant addition was helpful to enhance the mechanical properties.

4. Further research will be conducted on the wear properties of $\mathrm{Ni}-\mathrm{Cr}$ based composites with the single, dual and multiple solid lubricants in order to achieve a composite with excellent mechanical as well as wear properties.

\section{Acknowledgement}

The author would like to thank UNIMAS DPP grant for providing financial support of this research work.

\section{References}

[1] M. B. Peterson, S. LI, and S. F. Murray, "Wearresisting Oxide Films for $900^{\circ} \mathrm{C}$," J.Mater.Sci.Technol, 13, 99-106, (1997). 
[2] K. Miyoshi, "Solid Lubricants and Coatings for Extreme Environments : State-of-the-Art Survey," (2007).

[3] A. Vadiraj, M. Kamaraj, and V. S. Sreenivasan, "Effect of solid lubricants on friction and wear behaviour of alloyed gray cast iron," 37, 569577, (2012).

[4] S. Huang, Y. Feng, K. Ding, G. Qian, H. Liu, and Y. Wang, "Friction and wear properties of $\mathrm{Cu}-$ based self-lubricating," Acta Metall. Sin., 25, 391-400,(2012).

[5] M. Suzuki, M. Moriyama, M. Nishimura, and M. Hasegawa, "Friction and wear of self-lubricating composites at temperatures to $450{ }^{\circ} \mathrm{C}$ in vacuum," Wear, 162-164, 471-479, ( 1993).

[6] Q. Bi, S. Zhu, and W. Liu, "High Temperature Self-Lubricating Materials," in Tribology in Engineering, (2013).

[7] M. Xue, "High temperature oxidation and wear behaviour of powder metallurgically developed Ni-Cr-W-Al-Ti-MoS2 composite," Sci. York, 16, 111-115, (2009).

[8] C. H. Ding, P. L. Li, G. Ran, Y. W. Tian, and J. N. Zhou, "Tribological property of selflubricating PM304 composite," Wear, 262, 575581, (2007).

[9] E. Y. Liu, Y. M. Gao, W. Z. Wang, X. L. Zhang, X. Wang, G. W. Yi, and J. H. Jia, "Effect of the synergetic action on tribological characteristics of Ni-based composites containing multiplelubricants," Tribol. Lett., 47, 399-408, (2012).

[10] C. Suiyuan, W. Jing, L. Yijie, L. Jing, and L. Changsheng, "Synthesis of New Cu-based Selflubricating Composites with Great Mechanical Properties," J. Compos. Mater., 45, 51-63, (2010).

[11] L. F. Güleryüz, S. Ozan, R. İpek, and D. Uzunsoy, "Production of B $4 \mathrm{C} p$ reinforced magnesium metal matrix composites by powder metallurgy," 1, 51-58, (2012).

[12] X. Dangsheng, "Lubrication behavior of Ni-Crbased alloys containing MoS2 at high temperature," Wear, 251, 1094-1099, (2001).

[13] F. Li, J. Cheng, Z. Qiao, J. Ma, S. Zhu, L. Fu, J. Yang, and W. Liu, "A Nickel-Alloy-Based HighTemperature Self-Lubricating Composite with Simultaneously Superior Lubricity and High Strength," Tribol. Lett., 49, 573-577, (2013). 